\title{
Successful Treatment of Diffuse Large B-cell Lymphoma Following Waldenström's Macroglobulinemia with CHOP Chemotherapy Followed by Combination Therapy of CHOP with Rituximab
}

\author{
Keita Uchino, Hidekazu SAmEshima, Toshihiro MiYamoto, \\ Tadafumi IInO, Koji KATO, Hideho HenZAN, Ken-ichi AOKI, \\ Koji NAGAFuJI, Hisashi Gondo and Mine HaRADA
}

\begin{abstract}
We report a 72-year-old man with Waldenström's macroglobulinemia (WM) in whom diffuse large B-cell lymphoma (DLCL) occurred 17 years after the diagnosis of WM. The malignant cells of both DLCL and WM expressed CD20 on their surface. CHOP plus anti-CD20 monoclonal antibody, rituximab, were effective for both diseases, and the patient remains disease-free 17 months later.
\end{abstract}

(Internal Medicine 43: 131-134, 2004)

Key words: macroglobulinemia, diffuse large B-cell lymphoma, rituximab, CHOP

\section{Introduction}

Waldenström's macroglobulinemia (WM) is a slowly progressive, low-grade B-cell lymphoplasmacytic lymphoma characterized by a post-germinal center phenotype such as CD20 and CD19. Although the clinical course of WM is usually indolent, the disease may rarely transform into a more aggressive B-cell lymphoma, which is thought to have a very poor prognosis despite intensified treatment (1). Recently, there is evidence that treatment with the anti-CD20 monoclonal antibody, rituximab, is effective in patients with CD20 ${ }^{+}$B-cell lymphoma (2) and in WM (3). We report a case of WM in whom $\mathrm{CD} 20^{+}$diffuse-large B-cell lymphoma developed and was successfully treated with CHOP chemotherapy plus rituximab.

\section{Case Report}

A 55-year-old Japanese man was first admitted to a nearby hospital because of general fatigue and headache in October 1984. A diagnosis of WM was made based on the presence of monoclonal IgM protein of $52.9 \mathrm{~g} / \mathrm{l}$ and proliferation of lymphoplasmacytoid cells in the marrow. Treatment was successful with plasmapheresis for hyperviscosity syndrome. The patient had improved and was treated with monthly plasmapheresis without any cytotoxic chemotherapy over a period of 17 years. In December 2001, he complained of the persistent fever. In January 2002, he was referred to our hospital on emergency because of the hypovolemic shock due to severe dehydration by persistent fever. On physical examination, he had massive splenomegaly. He had no signs of hyperviscosity syndrome. The hemoglobin concentration was $12.7 \mathrm{~g} / \mathrm{dl}$, platelet count 230 $\times 10^{9} / l$, and white blood cell count $6.51 \times 10^{9} / l$ with $58 \%$ neutrophils, 12\% monocytes, 24\% lymphocytes, and $6 \%$ eosinophils. Serum lactate dehydrogenase level increased to $571 \mathrm{U} / l$ (normal range, 119-229 U/l). A bone marrow aspirate was normocellular with $39 \%$ abnormal lymphoplasmacytoid cells, which was positive for CD19, CD20, CD38, HLA-DR, surface IgM (sIgM), and kappa light chain (Fig. 1A). Serum electrophoresis revealed an increased level of IgM $(23.1 \mathrm{~g} / l)$ and monoclonal IgM kappa. Computed tomographic (CT) scans of the abdomen showed marked enlargement of the spleen and a retroperitoneal mass $(5 \times 5 \mathrm{~cm})$ (Fig. 2A). Since the patient presented with marked deterioration in performance status, aspiration needle biopsy of the retroperitoneal mass was chosen instead of the laparatomy. The specimens showed diffuse proliferation of abnormal

From the Medicine and Biosystemic Science, Kyushu University Graduate School of Medical Sciences, Fukuoka

Received for publication May 16, 2003; Accepted for publication October 9, 2003

Reprint requests should be addressed to Dr. Toshihiro Miyamoto, the Medicine and Biosystemic Science, Kyushu University Graduate School of Medical Sciences, 3-1-1 Maidashi, Higashi-ku, Fukuoka 812-8582 


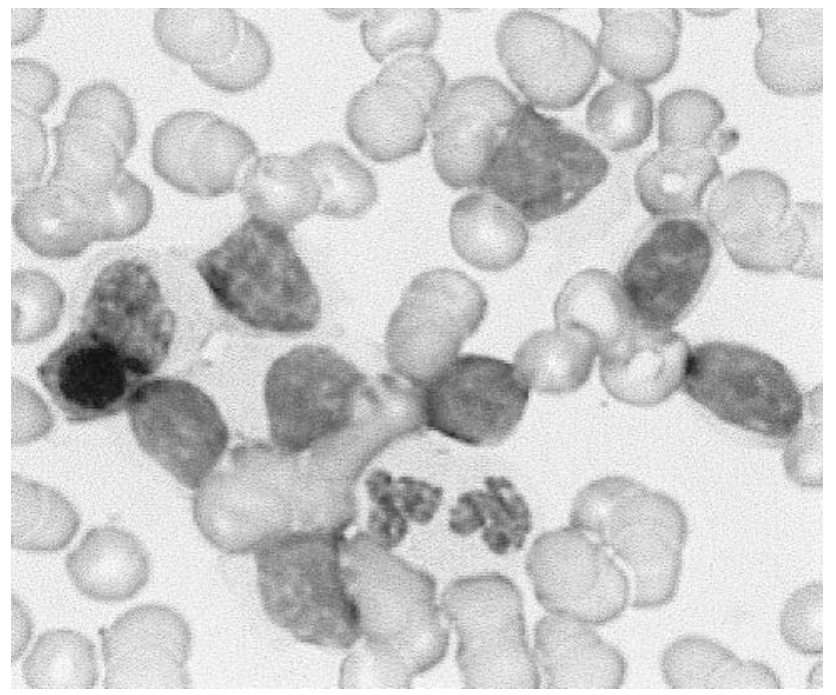

A

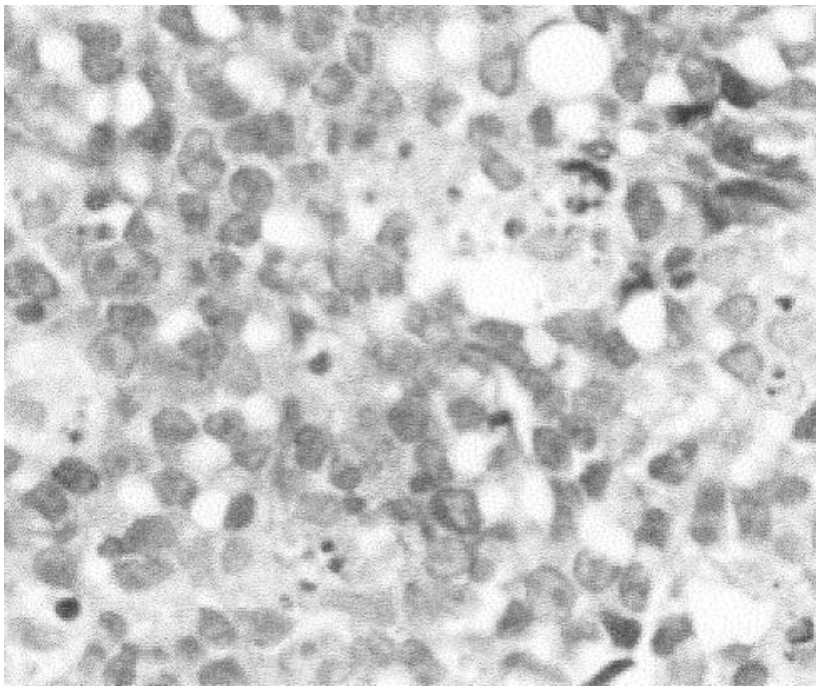

B

Figure 1. Bone marrow aspirate $($ May-Giemsa stain $\times 400)($ A) and retroperitoneal mass biopsy specimen showing a diffuse proliferation of large lymphoid cells $(\mathrm{HE}, \times 160)(B)$ on admission.

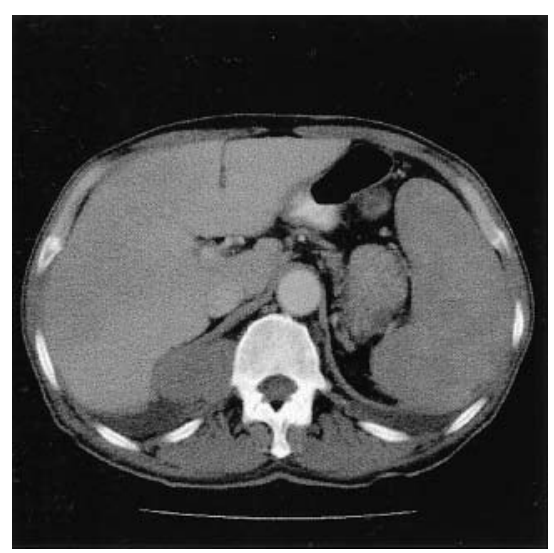

A

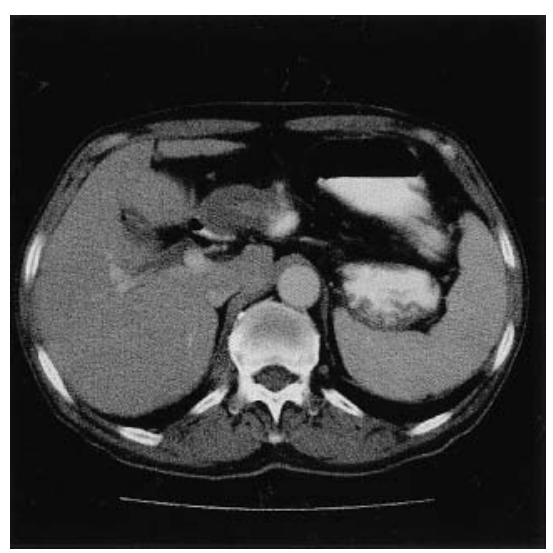

B

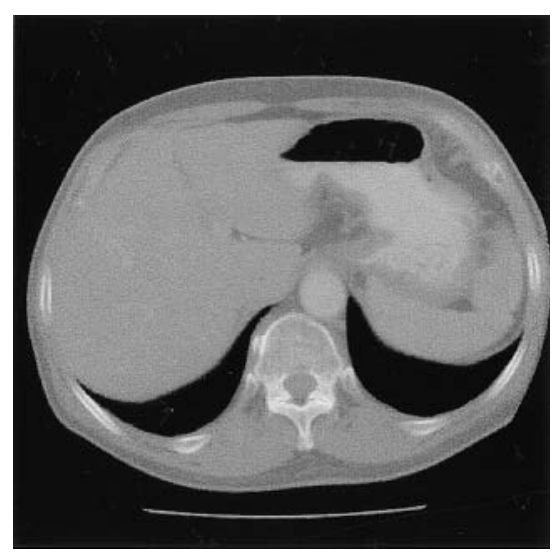

C

Figure 2. Computed tomographic scans of the abdomen, showing marked splenomegaly and retroperitoneal mass at presentation (A). Treatment with four courses of CHOP was effective with the reduction of the spleen and retroperitoneal mass (B). Disappearance of the retroperitoneal mass after treatment with 6 courses of CHOP plus rituximab (C).

large lymphoid cells that were immunohistochemically positive for CD20, sIgM, and kappa light chain, consistent with a diffuse large B-cell lymphoma (DLCL) (Fig. 1B). Thus, the patient had stage IVB disease of DLCL with high risk on the International Prognostic Index (4).

Initially, the patient was treated with standard $\mathrm{CHOP}$ regimen consisting of $50 \mathrm{mg} / \mathrm{m}^{2}$ of doxorubicin intravenously (iv) on day $1,1.4 \mathrm{mg} / \mathrm{m}^{2}$ of vincristine iv on day $1,750 \mathrm{mg} /$ $\mathrm{m}^{2}$ of cyclophosphamide iv on day 1 and $40 \mathrm{mg} / \mathrm{m}^{2}$ of prednisolone orally for five days every three weeks. After four courses of CHOP, treatment was effective with resolution of fever, decrease of serum IgM values (from $23.1 \mathrm{~g} / \mathrm{l}$ to
$9.0 \mathrm{~g} / l)$ and reduction of the abnormal lymphoplasmacytoid cells in marrow (from 39\% to 8\%) (Fig. 3). His spleen was also decreased in size from $12 \mathrm{~cm}$ to a palpable edge below the costal margin. CT scans showed a significant regression of retroperitoneal mass (from $5 \times 5 \mathrm{~cm}$ to $1 \times 1 \mathrm{~cm}$; $96 \%$ decrease in the sum of the products of the greatest diameters (SPD)). A bone marrow aspirate showed $8 \%$ lymphoplasmacytoid cells, and no infiltrate of lymphoma cells. Thus, this patient was assessed as attaining a complete response (CR) on the criteria of International Workshop (5). However, the residual mass still remained at the retroperitonum (Fig. 2B), and several reports have stated that the prognosis of DLCL 


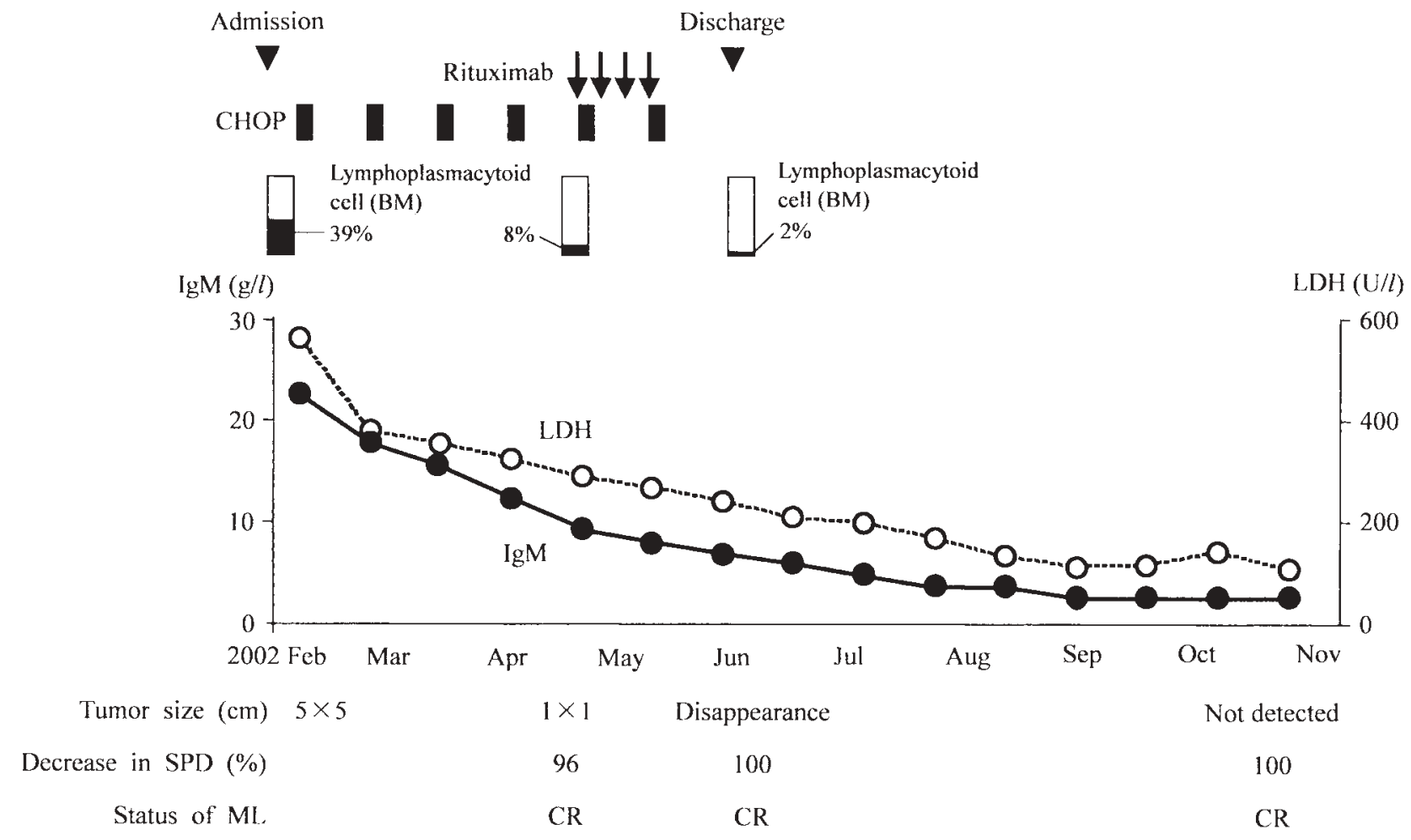

Figure 3. Clinical course of the patient. Decrease in sum of the products of the greatest diameters (SPD) of the retroperitoneal mass was evaluated by CT scans, and status of malignant lymphoma (ML) was assessed based on the criteria of International Workshop (5) on the clinical course. BM: bone marrow, CR: complete remission.

from WM is very poor despite cytotoxic chemotherapy $(1$, 6-10). Thus, we conducted a trial of treatment with rituximab plus the remaining courses of CHOP. The patient received rituximab, at a dose of $375 \mathrm{mg} / \mathrm{m}^{2}$ from day 1 of the 5 th course of CHOP. He was treated every week for a further 3 courses of rituximab and the 6th course of CHOP. After treatment with 6 courses of CHOP and rituximab therapy, CT scans showed that the retroperitoneal mass had completely disappeared and the spleen was decreased to the normal size (100\% decrease in SPD) (Fig. 2C). A bone marrow examination showed $2 \%$ lymphoplasmacytoid cells, and IgM was reduced to the normal range (Fig. 3). These examinations revealed the patient maintained CR for both DLCL and WM. At this time, 17 months after the diagnosis of DLCL, he is disease-free without any treatment, for both WM and DLCL.

To elucidate the clonal origin of DLCL and WM, DNA was extracted from the biopsy specimens of the retroperitoneal mass and the bone marrow, and amplification of the rearranged immunoglobulin heavy chain gene was performed by polymerase chain reaction (PCR). A single rearranged band was documented in the PCR analysis of bone marrow. PCR amplification was unsuccessful in the biopsy specimen because of degraded DNA.

\section{Discussion}

$\mathrm{WM}$ is an incurable, but generally indolent and slowly progressive low-grade lymphoplasmacytic lymphoma. The median survival of patients with WM averages 5 years (1113). Although the incidence is relatively low, chronic lymphoproliferative disorders can transform into a more immature and aggressive B-cell malignancy from a welldifferentiated one. One representative example is Richter's syndrome, an aggressive DLCL arising in patients with chronic lymphocytic leukemia (14). Similarly, patients with WM can undergo transformation into DLCL, and the prognosis of DLCL from WM is very poor, despite cytotoxic chemotherapy; the median survival was reported to be only 2 months (1, 6-10). Accordingly, intensified chemotherapy supported with autologous or allogeneic stem cell transplantation is necessary for this disease. However, these therapies would not be well tolerated by the majority of patients with WM because WM occurs at a median age of 63 years.

Since the chimeric anti-CD20 monoclonal antibody, rituximab, has shown efficacy in the treatment of indolent lymphoma (2), rituximab alone or in combination with other agents has been evaluated in many other B-cell lymphoproliferative disorders. Recently, a randomized study demonstrated that $\mathrm{CHOP}$ plus rituximab significantly 
increases the rate of complete response and improves overall survival in elderly patients with DLCL compared with CHOP alone (15). An encouraging result has also shown that rituximab is active in patients with WM (3). In these trials, rituximab was well tolerated by elderly patients, and the addition of rituximab to CHOP did not increase toxicity. Based on these data, $\mathrm{CHOP}$ plus rituximab was employed for the present patient since the malignant cells of both DLCL and WM expressed CD20 on their surface. Recently, however, tumor lysis syndrome (TLS) resulting from rituximab has been reported in patients with B-cell malignancies (16), especially leading to a sudden rise in serum IgM and viscosity levels in some WM patients (17). The present patient did not develop TLS because of the well-controlled IgM levels by the repeated courses of plasmapheresis before admission and the reduction of WM cells by the preceding four courses of $\mathrm{CHOP}$ before rituximab therapy. Therefore serum IgM levels and symptoms of hyperviscosity should be closely monitored for WM patients undergoing rituximab therapy.

We could not prove whether or not WM and DLCL in the present patient shared a common clonal origin. In this case, the lymphoma cells carried sIgM of the same isotype and the same light chain type as those detected on the WM cells, suggesting that DLCL might have been derived from the same clone. However, Chubachi et al (7) and Kogawa et al (10) reported that an independent clone was responsible for the development of DLCL in their patients with WM. These observations raise the possibility that DLCL is a second neoplasm arising from several risk factors associated with WM, such as destruction of the immune surveillance system, advanced age, and cytotoxic drugs. Treatment of such patients with chemotherapy plus rituximab may be feasible and effective when CD20 is expressed on both WM and DLCL cells.

\section{References}

1) Garcia R, Hernandez JM, Caballero MD, Gonzalez M, San Miguel JF. Immunoblastic lymphoma and associated non-lymphoid malignancies following two cases of Waldenstrom's macroglobulinemia. A review of the literature. Eur J Haematol 50: 299-301, 1993.

2) Maloney DG, Grillo-Lopez AJ, White CA, et al. IDEC-C2B8 (Rituximab) anti-CD20 monoclonal antibody therapy in patients with relapsed low-grade non-Hodgkin's lymphoma. Blood 90: 2188-2195, 1997.
3) Dimopoulos MA, Zervas C, Zomas A, et al. Treatment of Waldenstrom's macroglobulinemia with rituximab. J Clin Oncol 20: 2327-2333, 2002.

4) A predictive model for aggressive non-Hodgkin's lymphoma. The International Non-Hodgkin's Lymphoma Prognostic Factors Project. N Engl J Med 329: 987-994, 1993.

5) Cheson BD, Horning SJ, Coiffier B, et al. Report of an international workshop to standardize response criteria for non-Hodgkin's lymphomas. NCI Sponsored International Working Group. J Clin Oncol 17: 1244-1253, 1999 (Published erratum in J Clin Oncol 18: 2351, 2000).

6) Harousseau JL, Flandrin G, Tricot G, Brouet JC, Seligmann M, Bernard J. Malignant lymphoma supervening in chronic lymphocytic leukemia and related disorders. Richter's syndrome: a study of 25 cases. Cancer 48: 1302-1308, 1981.

7) Chubachi A, Ohtani H, Sakuyama M, et al. Diffuse large cell lymphoma occurring in a patient with Waldenstrom's macroglobulinemia. Evidence for the two different clones in Richter's syndrome. Cancer 68: 781-785, 1991.

8) Diebold J, Molina T, Tissier F, le Tourneau A, Audouin J. Waldenstrom's macroglobulinemia is a biological syndrome which may occur during the evolution of different types of low grade B cell lymphoma. Leukemia 13: 1637-1638, 1999.

9) Marinella MA, Kim MH, Anderson MM. Waldenstrom's macroglobulinemia transformed into immunoblastic lymphoma presenting with malignant ascites. Am J Hematol 51: 249-250, 1996.

10) Kogawa $K$, Sato $T$, Inohara $T$, et al. Waldenstrom macroglobulinemia with diffuse large B-cell lymphoma that developed from a different Bcell clone. Rinsho Ketsueki 40: 1116-1123, 1999 (in Japanese).

11) Kyrtsonis MC, Vassilakopoulos TP, Angelopoulou MK, et al. Waldenstrom's macroglobulinemia: clinical course and prognostic factors in 60 patients. Experience from a single hematology unit. Ann Hematol 80: 722-727, 2001 (Published erratum in Ann Hematol 81: 124, 2002).

12) Garcia-Sanz R, Montoto S, Torrequebrada A, et al. Waldenstrom macroglobulinaemia: presenting features and outcome in a series with 217 cases. Br J Haematol 115: 575-582, 2001.

13) Gertz MA. Waldenstrom's macroglobulinemia: a review of therapy. Leuk Lymphoma 43: 1517-1526, 2002.

14) Richter MN. Generalized reticular cell sarcoma of lymph nodes associated with lymphatic leukemia. Am J Pathol 4: 285-292, 1928.

15) Coiffier B, Lepage E, Briere J, et al. CHOP chemotherapy plus rituximab compared with $\mathrm{CHOP}$ alone in elderly patients with diffuse large-B-cell lymphoma. N Engl J Med 346: 235-242, 2002.

16) Yang H, Rosove MH, Figlin RA. Tumor lysis syndrome occurring after the administration of rituximab in lymphoproliferative disorders: highgrade non-Hodgkin's lymphoma and chronic lymphocytic leukemia. Am J Hematol 62: 247-250, 1999.

17) Gertz MA, Anagnostopoulos A, Anderson K, et al. Treatment recommendations in Waldenstrom's macroglobulinemia: consensus panel recommendations from the Second International Workshop on Waldenstrom's Macroglobulinemia. Semin Oncol 30: 121-126, 2003. 